AperTO - Archivio Istituzionale Open Access dell'Università di Torino

\title{
Approximation of $\mathbf{2 d}$ and $\mathbf{3 d}$ models of chemotactic cell movement in vasculogenesis
}

\section{This is the author's manuscript}

Original Citation:

\section{Availability:}

This version is available http://hdl.handle.net/2318/93505

since 2017-03-13T10:52:38Z

Publisher:

Springer

Published version:

DOI:10.1007/978-3-540-44446-6_15

Terms of use:

Open Access

Anyone can freely access the full text of works made available as "Open Access". Works made available under a Creative Commons license can be used according to the terms and conditions of said license. Use of all other works requires consent of the right holder (author or publisher) if not exempted from copyright protection by the applicable law. 


\title{
Approximation of 2D and 3D Models of Chemotactic Cell Movement in Vasculogenesis
}

\author{
Fausto Cavalli ${ }^{1}$, Andrea Gamba ${ }^{2}$, Giovanni Naldi $^{1}$, and Matteo Semplice ${ }^{1}$ \\ 1 Dipartimento di Matematica, Università di Milano, via Saldini 50, 20133 Milano, \\ Italy $\{$ cavalli, naldi, semplice\}@mat.unimi.it \\ 2 Dipartimento di Matematica, Politecnico di Torino, Corso Duca degli Abruzzi \\ 24, 10129 Torino, Italy \\ andrea.gamba@polito.it
}

Cell migration plays a central role in a wide variety of biological phenomena. In the case of chemotaxis, cells (or an organism) move in response to a chemical gradient. Chemotaxis underlies many events during embryo development and in the adult body. An understanding of chemotaxis is not only gained through laboratory experiments but also through the analysis of model systems, which often are more amenable to manipulation. This work is concerned with the relaxation schemes for the numerical approximation of a $2 \mathrm{D}$ and $3 \mathrm{D}$ model for cell movement driven by chemotaxis. More precisely, we consider models arising in the description of blood vessels formation and network formation starting from a random cell distribution.

\section{A Model for Vasculogenesis}

\subsection{Brief Biological Background}

It is well known that the embryonic heart and vasculature are the first organs which start functioning with largely the same main purpose as in the adult: blood transport. The origin and assembly of embryonic blood vessels not only involve multiple sources of precusor cells, but are also influenced by various combinations of proliferation, migration, differentiation, competition between cell-cell and cell-matrix interactions [7, 15, 27, 28].

The process of formation of this initial vascular network is called vasculogenesis. As the organism develops, subsequent growth and remodelling of the vascular network occur mainly via angiogenesis, whereby vessels sprout from existing vessels into surrounding tissues [20, 22].

In this work we consider a numerical simulation of a model of the vasculogenesis phenomenon in order to describe the de novo blood vessel formation from the mesoderm. The vasculogenetic process is driven by the recruitment 
of undifferentiated mesodermal cells in the embryonic body to the endothelial lineage and the de novo assembly of such cells into blood vessels. Results from a number of technical advances and studies (for a review see, for example, $[7,21])$ have defined the following as essential steps in the process:

1. the birth of angioblasts (the endothelial cell precursors);

2. the aggregation of angioblasts;

3. the elongation of angioblasts into chord-like structures;

4. the organization of isolated vascular segments into a capillary-like network and, concomitant with this step, their endothelialization and lumenization.

It is also important to note that blood vessels formed by vasculogenesis are initially free of smooth muscle cells, pericytes, and other associated cells; they may remain as nascent endothelial tubes for a considerable period of time.

In order to study and identify the factors influencing blood vessel formation, several mathematical models have been presented, see for example $[4,8,14,26,16]$. In this work we consider a mathematical model that describes biochemical interactions during blood vessel formation. The model consists of a system of non-linear partial differential equations and is based on the theory presented by Gamba et al. in [9] and by Serini et al. in [23].

\subsection{Mathematical Model}

As recent experiments (see [3]) show, the process of formation of a vascular network starting from a distribution of seeded cells can be tracked. The motion of each cell appears to be directed towards the areas of high concentration of cells. This fact suggests that chemotactic factors play a role in guiding cell motion. In chemotaxis phenomena, motile cells sense and respond directionally to chemical gradients due to some biochemical factor.

Here the cell population is described by a continuous density $n(\mathbf{x}, t)$, where $\mathbf{x} \in \mathbb{R}^{d}(d=2,3)$ is the space variable, while $t \geq 0$ is the time variable. The population density moves with velocities $\mathbf{v}(\mathbf{x}, t)$, that are triggered either by chemical gradients of a soluble factor or by random motion. The chemoattractant is described by a scalar chemical concentration field $c(\mathbf{x}, t)$. Moreover, the chemical factor is supposed to be released by the cells themselves, diffuse, and degrade in finite time, in agreement with experimental observations. These assumptions give rise to the following system (see [9]):

$$
\begin{aligned}
& \frac{\partial n}{\partial t}+\nabla \cdot(n \mathbf{v})=0 \\
& \frac{\partial \mathbf{v}}{\partial t}+\mathbf{v} \cdot \nabla \mathbf{v}=\mu(c) \nabla c-\nabla p(n)-\beta \mathbf{v} \\
& \frac{\partial c}{\partial t}=D \Delta c+g(n, c)-\frac{c}{\tau}
\end{aligned}
$$


Here $\mu(c)$ measures the cell response to the chemotactic factor, while $D$ and $\tau$ are respectively the diffusion coefficient and the characteristic degradation time of the soluble chemoattractant. Finally the function $g(n, c)$ determines the rate of release of the chemical factor.

A simple model may be obtained by assuming a constant cell sensitivity $\mu(c)=\mu_{0}$ and that the release of the chemoattractant is linearly proportional to the cell density: $g(n, c)=\alpha n$, with constant rate $\alpha>0$. A more realistic description may be obtained including saturation effects via non-linearities in the function $g(n, c)$ or by considering non-constant functions $\mu(c)$. (For the details, please refer to section 3.)

The term $\nabla p(n)$ is a density dependent pressure where $p(n)$ is zero for low densities, and increases for densities above a suitable threshold. This pressure is a phenomenological term which tries to model short range interaction between cells and the fact that cells do not compenetrate and have some degree of rigidity. Finally, the friction term mimics the adhesion of the cells to the substrate.

Initial conditions are given as a set of uniform randomly distributed bumps in the density field, with zero velocities and zero concentration of the chemoattractant. Starting from these conditions, equations (1) are integrated numerically.

The two-dimensional model has a clear biological counterpart: it may be directly compared with "in vitro" experiments of self-organization of endothelial cells seeded on a matrigel plate that are observed to evolve forming a network-like structure (see [23] and references therein). Quantitative comparisons may be achieved by studying the percolation properties of the network observable in the final state of $n(\mathbf{x})$ when the initial cell density is varied and by measuring the average vessel length in the final state (see [9]).

Since 1997, the biologists' community has become aware of the importance of the extracellular structures for the behaviour of the cells. M. Weawer at al., in their seminal paper [29], showed that a line of cancer cells has two completely different behaviours in a $2 \mathrm{D}$ and inside a $3 \mathrm{D}$ culture. Since then much work has been done in this direction, proving the need of three-dimensional cell cultures to reproduce more faithfully the biochemical activity of living beings (see the review papers $[1,6]$ ).

It is known that in the early stages of development almost all intraembryonic mesodermal tissues contain migrating endothelial precursors, which appear to be randomly scattered (see e.g. [5]). Therefore in the three dimensional version of our model (1) we use initial conditions that represent a randomly scattered distribution of cells. Thus, model (1) is a candidate to describe experiments of vasculogenetic processes, both "in vivo" and in three dimensional cell cultures. 


\section{Relaxation Approximation}

In order to solve numerically system (1), we consider schemes which are based on a suitable relaxation approximation. Such a scheme permits to reduce nonlinear second order equations or quasilinear conservation laws to first order semi-linear hyperbolic systems with stiff terms. In the case of approximation of a scalar conservation law

$$
\frac{\partial u}{\partial t}+\frac{\partial}{\partial x} f(u)=0
$$

Jin and Xin [12] have proposed the following system

$$
\begin{aligned}
& \frac{\partial u}{\partial t}+\frac{\partial j}{\partial x}=0 \\
& \frac{\partial j}{\partial t}+a \frac{\partial u}{\partial x}=-\frac{1}{\epsilon}(j-f(u)),
\end{aligned}
$$

where $j$ plays the role of a physical flux, $\epsilon$ is a small positive parameter, called relaxation time, and $a$ is a suitable positive constant. The first order approximation of the conservation law (3) is

$$
\frac{\partial u}{\partial t}+\frac{\partial}{\partial x} f(u)=\epsilon \frac{\partial}{\partial x}\left(\left(a-f^{\prime}(u)^{2}\right) \frac{\partial u}{\partial x}\right)
$$

which can be derived using the Chapman-Enskog expansion. It is also clear that (4) is dissipative, provided that the subcharacteristic condition $a>f^{\prime}(u)^{2}$ is satisfied. We would expect that appropriate numerical discretization of the relaxation system (3) yields accurate approximation to the original equation (2) when the relaxation parameter $\epsilon$ is sufficiently small.

In view of its numerical approximation, the main advantage of the relaxation system (3) over the original equation (2) lies in the linear structure of the characteristic fields and in the localized low order term. In particular this linear structure avoids the use of time consuming Riemann solvers. Moreover, proper implicit time discretizations can be exploited to overcome the stability constraints due to the stiffness and to avoid the use of non-linear solvers.

In recent years several relaxation approximations to partial differential equations of various type have been proposed, from kinetic schemes for gas dynamics to general relaxation schemes for conservation laws and diffusive relaxation schemes for convection-diffusion and reaction-diffusion problems $[2,13,19]$. For example, let's consider the following one-dimensional equation

$$
\frac{\partial u}{\partial t}+\frac{\partial}{\partial x} f(u)=\frac{\partial^{2}}{\partial x^{2}} p(u)
$$

where $p$ and $f$ are given smooth functions such that $p(0) \geq 0$ and $p^{\prime}(u)>0$. By introducing a new variable $j$, one can couple $j$ and $u$ in the following semi-linear hyperbolic system: 


$$
\begin{aligned}
& \frac{\partial u}{\partial t}+\frac{\partial j}{\partial x}=0 \\
& \frac{\partial j}{\partial t}+\frac{1}{\epsilon} \frac{\partial u}{\partial x}=-\frac{1}{\epsilon} k(u)(j-f(u)) .
\end{aligned}
$$

Here $\epsilon$ is the relaxation parameter and $k(u)=p^{\prime}(u)^{-1}$. As usual, when $\epsilon \ll 1$, system (5) is said to be stiff. In order to consider also degenerate diffusion problems, a different relaxation system can be introduced with a third variable $w$,

$$
\begin{aligned}
& \frac{\partial u}{\partial t}+\frac{\partial j}{\partial x}=0 \\
& \frac{\partial j}{\partial t}+\frac{1}{\epsilon} \frac{\partial w}{\partial x}=-\frac{1}{\epsilon}(j-f(u)) \\
& \frac{\partial w}{\partial t}+a \frac{\partial j}{\partial x}=-\frac{1}{\epsilon}(w-p(u))
\end{aligned}
$$

with a suitable positive constant $a$ to control the stability condition.

The numerical passage from the relaxation system to the non-linear diffusion equation is realized by using semi-implicit or fully implicit time discretization combined with upwind and central differences in space. If we consider, as a prototype for the numerical scheme, the linear diffusion equation $\frac{\partial u}{\partial t}-\frac{\partial^{2} u}{\partial x^{2}}=0$, it sufficies to consider the following relaxation system (also called Maxwell-Cattaneo system)

$$
\begin{aligned}
& \frac{\partial u}{\partial t}+\frac{\partial j}{\partial x}=0 \\
& \frac{\partial j}{\partial t}+\frac{1}{\epsilon} \frac{\partial u}{\partial x}=-\frac{1}{\epsilon} j .
\end{aligned}
$$

In this simple case, an analysis of the drawbacks of the different numerical approaches is described in [17]. Let $\Delta t$ and $\Delta x$ be respectively the time step and the uniform grid spacing. The standard semi-implicit discretization in conservative form, for small values of $\epsilon$, leads to a modified equation with a numerical dissipation rate of order $\Delta x / 2 \epsilon$. Then, if $\Delta x \geq \epsilon$ the numerical dissipation will be comparable with, or dominate, the physical dissipation. We also have to solve in time the stiffness of this numerical dissipation term and hence the CFL condition is of the type $\Delta t \simeq \epsilon \Delta x$. Clearly, this is too restrictive near the parabolic regime $\epsilon \ll \Delta x$ where a condition of the type $\Delta t \simeq \Delta x^{2}$ is expected. A different upwind selection for the numerical flux which avoids unacceptable CFL condition and permits high order extension, is proposed in [17]. However the modified scheme was difficult to generalize to nonlinear diffusion. A more general and reliable numerical recipe is considered in $[13,18]$. The main idea consists in splitting the scales by rewriting the relaxation system as 


$$
\begin{aligned}
& \frac{\partial u}{\partial t}+\frac{\partial j}{\partial x}=0 \\
& \frac{\partial j}{\partial t}+\lambda(\epsilon) \frac{\partial u}{\partial x}=-\frac{1}{\epsilon}\left(j+(1-\epsilon \lambda(\epsilon)) \frac{\partial u}{\partial x}\right),
\end{aligned}
$$

where $\lambda(\epsilon)$ is such that $0 \leq \lambda(\epsilon) \leq 1 / \epsilon$. This restriction on $\lambda$ guarantees the positivity of both $\lambda$ and $(1-\epsilon \lambda)$. Now, as it is usually done for kinetic equations or hyperbolic systems with relaxation, (8) can be split into two subproblems:

$$
\begin{aligned}
& \frac{\partial u}{\partial t}+\frac{\partial j}{\partial x}=0 \\
& \frac{\partial j}{\partial t}+\lambda(\epsilon) \frac{\partial u}{\partial x}=0
\end{aligned}
$$

and

$$
\begin{aligned}
& \frac{\partial u}{\partial t}=0 \\
& \frac{\partial j}{\partial t}=-\frac{1}{\epsilon}\left(j+(1-\epsilon \lambda(\epsilon)) \frac{\partial u}{\partial x}\right) .
\end{aligned}
$$

We remark that the standard splitting does not work for (7) because, when $\epsilon \rightarrow 0$, we obtain the equilibrium equations

$$
j=0 \quad \text { and } \quad \frac{\partial u}{\partial x}=0
$$

that are inconsistent with the diffusive limit.

Summarizing, we can consider relaxation approximation both for conservation laws and for diffusion equations. Hence we can adopt the same approach for the whole model of vasculogenesis. In the case of multidimensions, a similar discretization can be applied to each space dimension [12, 13, 18]. Then, since the structure of the multidimensional diffusive relaxation system is similar to the 1D system, the numerical implementation for higher dimensional problems, based on dimensional splitting, is not much harder than for 1D problems.

Very fine grids have to be used, in order to resolve the details of the field $n(\mathbf{x}, t)$, which might have hundreds of small bumps, each representing a single cell. The computational cost may be reduced by using parallel computing: the semilinearity of relaxation systems, together with appropriate discretizations, gives rise to parallel algorithms with almost optimal scaling properties.

\section{Numerical Results}

As in $[9,23]$ we perform two-dimensional numerical simulations of model (1) on a square box with side of lentgh $L=1 \mathrm{~mm}$, with periodic boundary conditions. The relaxation system is numerically solved by considering the splitting 
scheme (9) for the pure convective step and the relaxation step. These steps are combined in a second order Runge Kutta scheme (see [11, 12]). For the numerical discretization of the transport step, in order to achieve an accuracy of $O\left(\Delta x^{2}\right)$, we use a second order TVD scheme with suitable slope limiter (see $[10,24])$.

Biochemical experiments suggests the values of $D=10^{-3} \mathrm{~mm}^{2} / \mathrm{sec}$ and $\tau=4000$ sec for the diffusion constant and the decay rate of the chemoattractant. We fix the other constant parameters by dimensional analysis and the consideration of the characteristic scales of the system. In particular, for the two-dimensional model, we choose: $\mu_{0}=10^{-11} \mathrm{~mm}^{4} / \mathrm{sec}^{3}, \alpha=1 \mathrm{sec}^{-1}$, $\beta=10^{-3} \sec ^{-1}$.

The pressure function is taken to be

$$
p(n)= \begin{cases}C_{p}\left(n-n_{0}\right)^{3} & n>n_{0} \\ 0 & n \leq n_{0}\end{cases}
$$

where $n_{0}=6.0$ and the areas where $n(\mathbf{x})<6$ (lighter gray in Figures 1, 2, 4 and 5) represent locations where network chords are thinner (one may see this effect on the plates of the "in vitro" experiments, as in Figure 3).

With this naive setting, we observe that the initial random distribution of cells evolve into a network that, however, represents only a transient state: eventually large blobs of matter arise. Figure 1 represents this situation: the cell density is shown at time $t=0,60$ and 150 minutes.
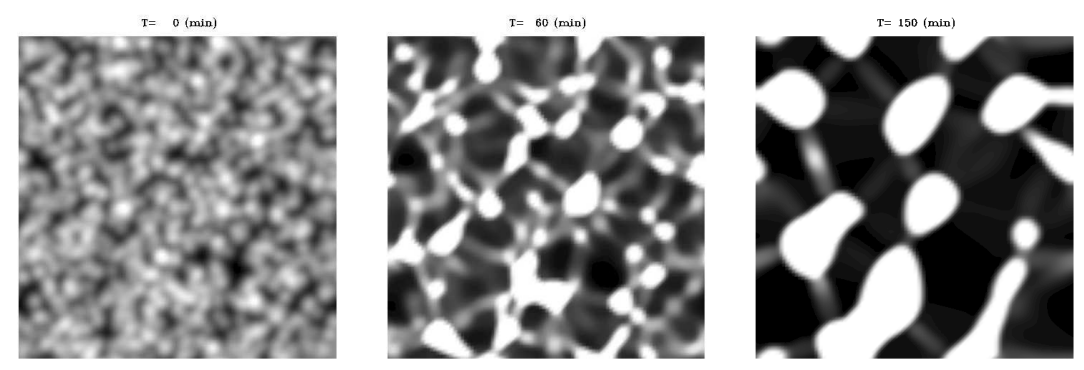

Fig. 1: Transient network state. With a linear emisison rate $g(n, c)=\alpha n$ and constant $\mu(c)=\mu_{0}$, the network state is only transient. These images plot the cell density distribution at time $t=0,60,150$ minutes. The initial distribution is random with a mean density of 200 cell $/ \mathrm{mm}^{2}$.

It is natural, from a biological point of view, to suppose that saturation effects play a role. One may think that, when the background concentration of chemoattractant is high, the cells react more weakly to its gradient or that they emit less chemoattractant when its concentration in the surrounding space is already significant. These effects may be incorporated in our model 
using a non-constant sensitivity $\mu(c)$, a non-linear emission rate $g(n, c)$, or both. We choose a threshold $c_{0}$ and then functions like

$$
\begin{aligned}
\mu(c) & =\mu_{0}\left(1-\tanh \left(c-c_{0}\right)\right) \\
g(n, c) & =\alpha n\left(1-\tanh \left(c-c_{0}\right)\right)
\end{aligned}
$$

The net effect is that the sensitivity of the cells and respectively their production of chemoattractant is strongly damped when the concentration $c$ reaches the threshold $c_{0}$. We did not observe a significant dependence on the exact form of the damping function, provided that it approximates a step function that is nonzero only when $c<c_{0}$. With the above choices we obtain a network state that is not transient any more: the system settles onto it (see Figures $2,4,5)$.

In Figures 2,4 and 5 we present the results obtained with $c_{0}=1000$. For comparison purposes, the initial random distrubution of cells is the same as in Figure 1 and it is not shown. Using (10a), we observe that the state reached at time $t=60 \mathrm{~min}$ is not significantly modified any more in the evolution (Figure $2)$. The density $n(\mathbf{x}, t)$ reaches a state that consists of blobs of matter linked by thinner chords, which is remarkably similar to the final state obtained in "in vitro" experiments (Figure 3). The numerical tests indicate that this network-like state is stable, unlike the case of Figure 1.
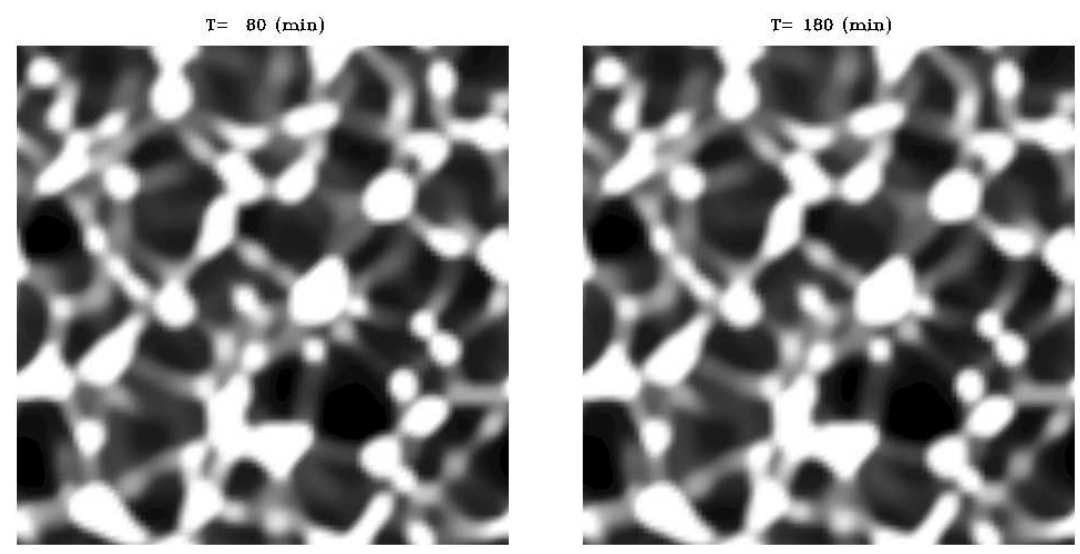

Fig. 2: Results of the numerical evolution of system (1), together with (10a) and linear $g(n, c)=\alpha n$. The images plot the cell density distribution at time $t=60,180$ minutes. The initial distribution is the same as in Figure 1.

On the other hand, (10b) and a constant $\mu(c)$, is less effective in bringing the system to a network-like state, but the final state presents thinner chords, 


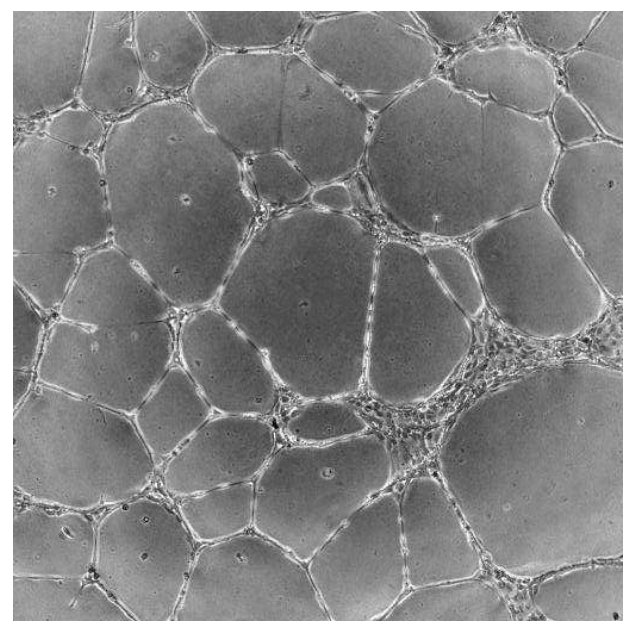

Fig. 3: In vitro experiment of self-organization of endothelial cells seeded on a matrigel plate of $2 \times 2 \mathrm{~mm}^{2}$. (Thanks to G. Serini, IRCC, Candiolo - Torino)

as is shown in Figure 4. This is to be expected since (10b) acts on equation (1c), where it competes with the decay term.
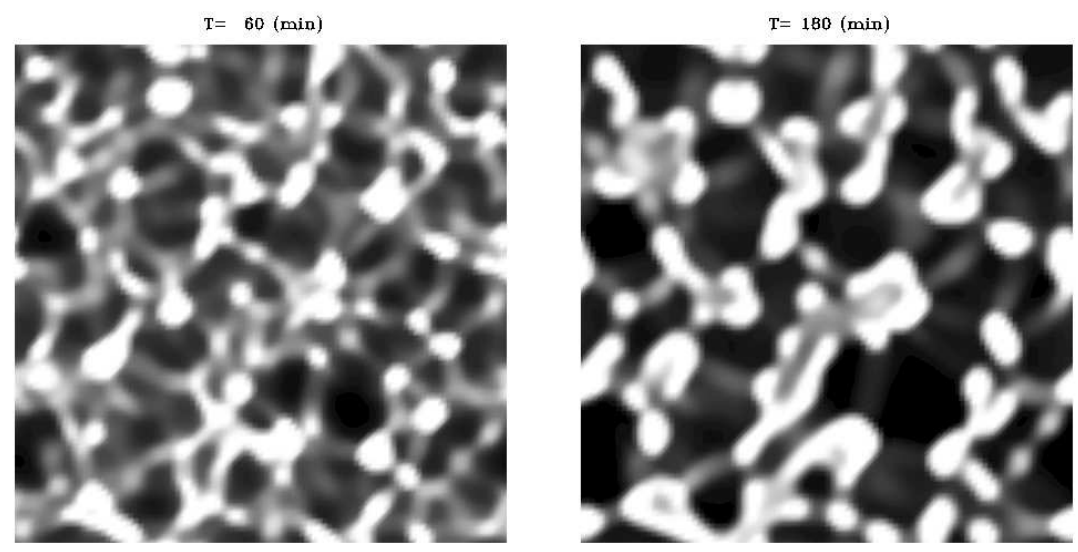

Fig. 4: Results of the numerical evolution of system (1), together with (10b) and constant $\mu(c)=\mu_{0}$. The images plot the cell density distribution at time $t=60,180$ minutes. The initial distribution is the same as in Figure 1.

Finally, Figure 5 shows that using both equations (10), with the same threshold $c_{0}$, the behaviour of Figure 2 prevails. 
We observe here that in Nature several effects are reasonably expected to concur to the stabilization of network structures. Along with saturation effects, mechanical interactions also produce network stabilization ([25]).
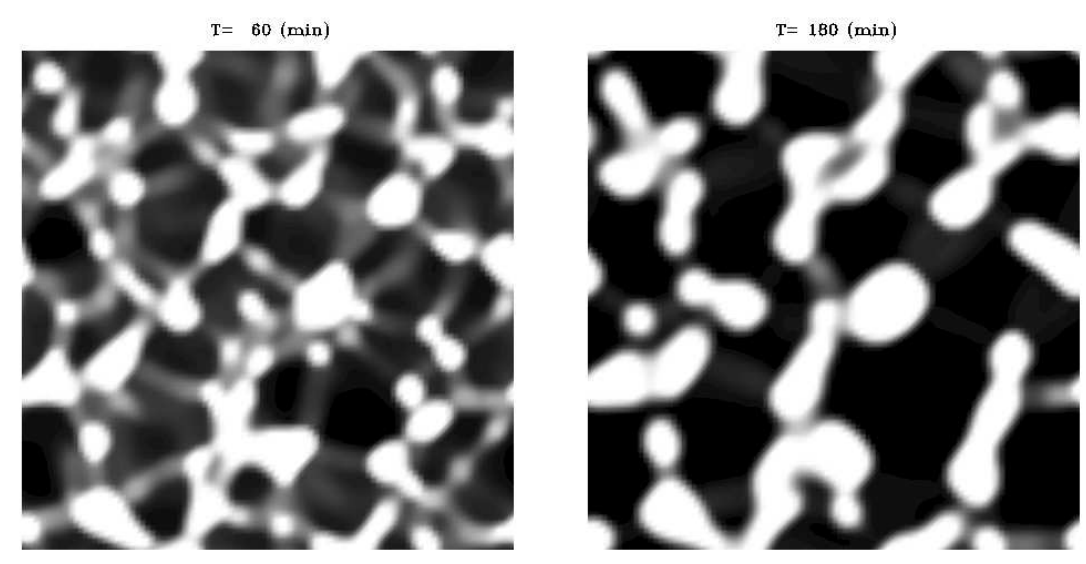

Fig. 5: Results of the numerical evolution of system (1), together with both (10a) and (10b). The images plot the cell density distribution at time $t=60,180$ minutes. The initial distribution is the same as in Figure 1.

In three dimensions we used the same parameter setting as in the twodimensional case. Due to the computational costs, the algorithm has been implemented on a high performance cluster for parallel computation installed at the Department of Mathematics of the University of Milano (see http://cluster.mat.unimi.it/).

We observed that, starting with an initial random distribution of cells, the cell density evolves towards a network-like state. Figure 6 shows this state: it is transient since, in this simulation, we used a linear function $g(n, c)$ and constant $\mu(c)$, but we expect that incorporating (10), the good results of the two-dimensional case should be reproduced here as well.

Our choice of a numerical algorithm that performs only linear operations and local function evaluations allows us to obtain very good scaling properties in the parallel implementation, as shown in Figure 7.

\section{Conclusions}

In oder to gain understanding of the role of chemotaxis in the self-organization of cells, we performed numerical simulations aimed at reproducing the case of chemotaxis-driven vasculogenesis in two and three space dimensions. Our 

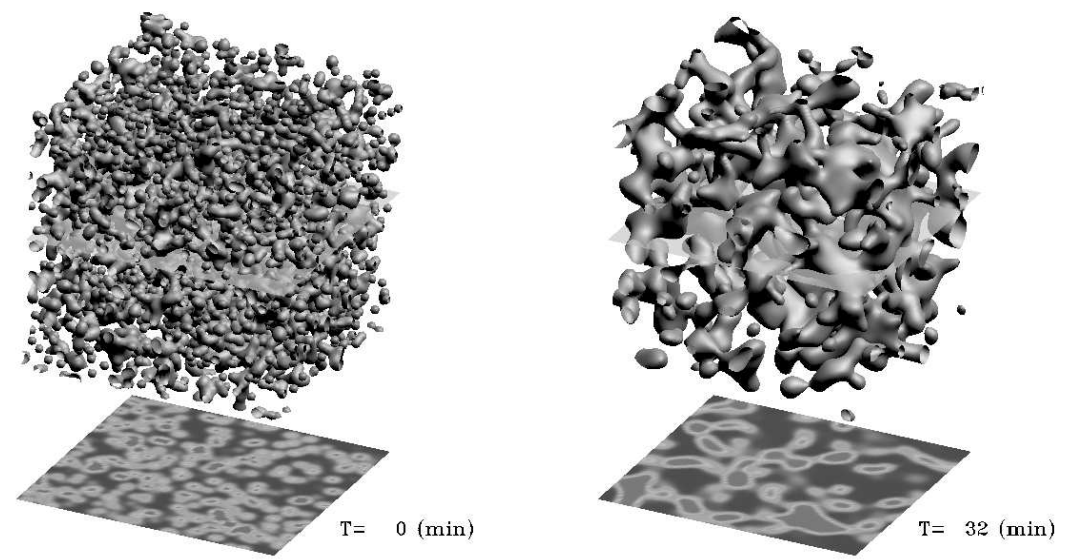

Fig. 6: Transient network state in 3D. These are the cell density distributions at time $t=0,32$ minutes. This was obtained with linear $g(c)$ and constant $\mu$. The images represent an isosurface plot together with a gray-level cross section at the indicated location.

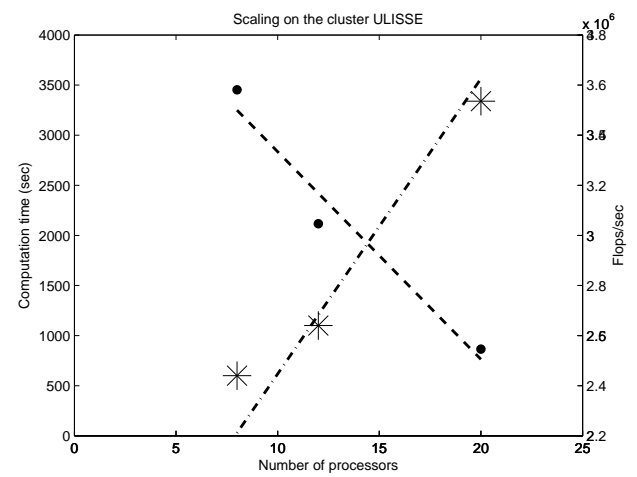

Fig. 7: Scaling of the 3D algorithm on the cluster ULISSE. The dots represent the execution time (in seconds) and the asterisks the Mflops/sec for our numerical algorithm. The dashed and dash-dot line are their respective linear interpolations.

model does not simulate the individual cells but rather a continuous spatial distribution of matter, whose bumps do represent the cells. This allows us to use the tools of partial differential equations to describe the mathematical model (1).

Proper spatial and temporal approximations (section 2) allow us to perform numerical simulations with an algorithm that performs only linear operations and local function evaluations without severe restrictions on the time step, despite the non-linearity of the original equations. This is also important 
for the implementation on parallel computers: the memory usage and the execution time of the algorithm decrease linearly with the number of processors and this allows us to reduce the computation time down to reasonable values.

The two-dimensional model has already proven to give results in good agreement with "in vitro" experiments of vasculogenesis. Our numerical tests indicate that the version that we present here possesses a stationary state; hence the model allows to investigate on the mechanisms that may play a role in bringing the biological system to stop its evolution when a proper prevascular network is formed. We proposed and compared mechanisms based on varying the sensitivity of the cells to the chemotactical signals and on varying the emission of the soluble chemoattractant.

Regarding the three dimensional model, we are currently working on the comparison of the numerical simulations with experimental data. Moreover we are working on the analysis of the numerical algorithms (stability and CFL condition for the non-linear relaxation).

\section{References}

1. A. Abbott: Biology's new dimension. Nature 424, 870-872 (2003)

2. D. Aregba-Driollet, R. Natalini, S. Q. Tang: Diffusive kinetic explicit schemes for nonlinear degenerate parabolic systems. Math. Comp. 73, 63-94, (2004)

3. P. Carmeliet: Mechanisms of angiogenesis and arteriogenesis. Nature Medicine 6, 389-395, (2000).

4. M. A. Chaplain: Mathematical modelling of angiogenesis. J. Neuro. 50, 37, (2000).

5. O. Cleaver and P. Krieg: Molecular mechanisms of vascular development. In R.P. Harvey and N. Rosenthal (eds): Heart development. Academic Press (1999), 221-252.

6. E. Cukierman, R. Pankov, D. R. Stevens, K. M. Yamada: Taking Cell-Matrix Adhesions to the Third Dimension. Science 294, 1708-1712 (2001)

7. C. J. Drake, J. E. Hungerford and C. D. Little: Morphogenesis of the First Blood Vessels. Ann. New York Acad. Sci. 857, 155-179, (1998).

8. E. A. Gaffney, K. Pugh, P. K. Maini and F. Arnold: Investigating a simple model of cutaneous wound healing angiogenesis. J. Math. Biol. 45, 337-374, (2002).

9. A. Gamba, D. Ambrosi, A. Coniglio, A. De Candia, S. Di Talia, E. Giraudo, G. Serini, L. Preziosi and F. Bussolino: Percolation, Morphogenesis, and Burgers Dynamics in Blood Vessels Formation Phys. Rev. Lett. 90, 118101 (2003).

10. A. Harten: High Resolution Schemes for Hyperbolic Conservation Laws. J. Comp. Phys. 49, 357-393, (1983).

11. S. Jin and Z. Xin: Runge-Kutta Methods for Hyperbolic Conservation Laws with Stiff Relaxation Terms. J. Comp. Phys. 122, 51-67, (1995).

12. S. Jin and Z. Xin: The Relaxation Schemes for Systems of Conservation Laws in Arbitrary Space Dimensions. Comm. Pure and Appl. Math. 48, 235-276, (1995).

13. S. Jin, L. Pareschi and G. Toscani: Diffusive Relaxation Schemes for DiscreteVelocity Kinetic Equations. SIAM J. Numer. Anal. 35, 2405-2439, (1998). 
14. H. A. Levine, B. D. Sleeman and M. Nilsen-Hamilton: Mathematical modeling of the onset of capillary formation initiating angiogenesis. J. Math. Biol. 42, 195-238, (2001).

15. C. D. Little: Vascular morphogenesis: in vivo, in vitro, in mente. Birkhäuser, Boston (1998).

16. R. MH Merks, A. Newman and J. A. Glazier: Cell-Oriented Modeling of in Vitro Capillary of Blood Vessel Growth. Lecture Notes in Computer Science 3305, 425-434, (2004).

17. G. Naldi and L. Pareschi: Numerical Schemes for Kinetic Equations in Diffusive Regimes. Appl. Math. Lett. 11, 29, (1998).

18. G. Naldi and L. Pareschi: Numerical Schemes for Hyperbolic Systems of Conservation Law with Stiff Diffusive Relaxation. SIAM J. Numer. Anal. 37, 12461270, (2000).

19. G. Naldi, L. Pareschi and G. Toscani: Relaxation schemes for PDEs and applications to fourth order diffusion equations. Surveys in Mathematics Applied to Industry 10, 315, (2002).

20. L. Pardanaud, F. Yassine, and F. Dieterlen-Lievre: Relationship between vasculogenesis, angiogenesis and haemopoiesis during avian ontogeny. Development 105, 473-485, (1989).

21. T. J. Poole, E. B. Finkelstein and C. M. Cox: The role of FGF and VEGF in angioblast induction and migration during vascular development. Dev. Dynam. 220, 1-17, (2001).

22. W. Risau, H. Sariola, H. G. Zerwes, J. Sasse, P. Ekblom, R. Kemler and T. Doetschmann: Vasculogenesis and angiogenesis in embryonic-stem-cell-derived embryoid bodies. Development 102, 471-478, (1988).

23. G. Serini, D. Ambrosi, E. Giraudo, L. Preziosi and F. Bussolino: Modeling the early stages of vascular network assembly. The EMBO Journal 22, 1771-1779, (2003).

24. P. R. Sweby: High Resolution Schemes Using Flux Limiters for Hyperbolic Conservation Laws. SIAM J. Num. Anal. 21, 995-1011, (1984).

25. A. Tosin, D. Ambrosi and L. Preziosi: Mechanics and chemotaxis in the morphogenesis of vascular networks. To appear in Bull. Mat. Biol. (2006)

26. S. Tong and F. Yuan: Numerical simulations of angiogenesis in the cornea. Microvasc. Res. 61, 14-27, (2001).

27. B. M. Weinstein: What guides early embryonic blood vessel formation? Dev. Dynam. 215, 2-17, (1999).

28. J. Wilting, S. Brand,H. Kurz and B. Christ: Development of the embryonic vascular system. Cell. Mol. Biol. Res. 41, 219-232, (1995).

29. Weaver VM, Petersen OW, Wang F, Larabell CA, Briand P, Damsky C, Bissell MJ: Reversion of the malignant phenotype of human breast cells in threedimensional culture and in vivo by integrin blocking antibodies. J. Cell. Biol. 137, 231-245 (1997). 
